\title{
QUEEN'S
UNIVERSITY
BELFAST
}

\section{Synthetic Lethal Targeting of ARID1A-Mutant Ovarian Clear Cell Tumors with Dasatinib}

Miller, R. E., Brough, R., Bajrami, I., Williamson, C. T., McDade, S., Campbell, J., Kigozi, A., Rafiq, R., Pemberton, H., Natrajan, R., Joel, J., Astley, H., Mahoney, C., Moore, J. D., Torrance, C., Gordan, J. D., Webber, J. T., Levin, R. S., Shokat, K. M., ... Ashworth, A. (2016). Synthetic Lethal Targeting of ARID1A-Mutant Ovarian Clear Cell Tumors with Dasatinib. Molecular Cancer Therapeutics, 15(7), 1472-1484. https://doi.org/10.1158/1535-7163.MCT-15-0554

Published in:

Molecular Cancer Therapeutics

Document Version:

Peer reviewed version

Queen's University Belfast - Research Portal:

Link to publication record in Queen's University Belfast Research Portal

Publisher rights

@2016 American Association for Cancer Research.

General rights

Copyright for the publications made accessible via the Queen's University Belfast Research Portal is retained by the author(s) and / or other copyright owners and it is a condition of accessing these publications that users recognise and abide by the legal requirements associated with these rights.

Take down policy

The Research Portal is Queen's institutional repository that provides access to Queen's research output. Every effort has been made to ensure that content in the Research Portal does not infringe any person's rights, or applicable UK laws. If you discover content in the Research Portal that you believe breaches copyright or violates any law, please contact openaccess@qub.ac.uk. 


\section{Synthetic Lethal Targeting of ARID1A Mutant Ovarian Clear Cell Tumours with Dasatinib}

Rowan E. Miller ${ }^{1,2}$, Rachel Brough ${ }^{1,2}$, Ilirjana Bajrami ${ }^{1,2}$, Chris T. Williamson ${ }^{1,2}$, Simon McDade $^{3}$, James Campbell ${ }^{1,2}$, Asha Kigozi ${ }^{1,2}$, Rumana Rafiq ${ }^{1,2}$, Helen Pemberton ${ }^{1,2}$, Rachel Natrajan ${ }^{2}$, Josephine Joel ${ }^{4}$, Holly Astley ${ }^{4}$, Claire Mahoney ${ }^{4}$, Jonathan D. Moore $^{4}$, Chris Torrance ${ }^{4,8}$, John D. Gordan ${ }^{5}$, James T Webber ${ }^{5}$, Rebecca S. Levin ${ }^{6}$, Kevan M. Shokat ${ }^{6,7}$, Sourav Bandyopadhyay ${ }^{5}$, Christopher J. Lord ${ }^{1,2 *}$ and Alan Ashworth ${ }^{1,2,9 *}$

${ }^{1}$ The CRUK Gene Function Laboratory and ${ }^{2}$ Breakthrough Breast Cancer Research Centre, The Institute of Cancer Research, London, SW3 6JB, UK

${ }^{3}$ Centre for Cancer Research and Cell Biology, Queen's University Belfast, University Road, Belfast, BT7 1NN, UK

${ }^{4}$ Horizon Discovery, 7100 Cambridge Research Park, Waterbeach, Cambridge, $\square$ CB25 9TL, UK

${ }^{5}$ UCSF Helen Diller Family Comprehensive Cancer Center, ${ }^{6}$ Cellular and Molecular Pharmacology and ${ }^{7}$ Howard Hughes Medical Institute, University of California, San Francisco, CA 94158, USA

${ }^{8}$ Current Address: PhoreMost, 23 Cambridge Science Park, Milton Road, Cambridge CB4 OEY, UK

${ }^{9}$ Current Address: UCSF Helen Diller Family Comprehensive Cancer Center, University of California, San Francisco, CA 94158, USA

Running title: Dasatinib/ARID1A synthetic lethality in OCCC

Keywords: ARID1A, Ovarian Clear Cell Carcinoma, Dasatinib, Synthetic Lethality

*Correspondence to: Christopher J. Lord, The Institute of Cancer Research, London, SW3 6JB, UK and Alan Ashworth, UCSF Helen Diller Family Comprehensive Cancer Center, University of California, San Francisco, CA 94158, USA

Email Chris.Lord@icr.ac.uk, Alan.Ashworth@ucsf.edu

Conflict of Interest disclosure: The authors have no conflicts of interest to declare. Word count (excluding references): 5143 (6470 including figure legends)

Total number of figures: 6 Total number of tables: 0 


\section{Financial Information}

This work was supported by grants to A. Ashworth and C.J. Lord from Cancer Research UK (C347/A8363), Breast Cancer Now and the European Union as part of the EUROCAN FP7 Programme (HEALTH-FP7-2010-260791). 


\section{Abstract}

New targeted approaches to ovarian clear cell carcinomas (OCCC) are needed, given the limited treatment options in this disease and the poor response to standard chemotherapy. Using a series of high-throughput cell based drug screens in OCCC tumor cell models, we have identified a synthetic lethal (SL) interaction between the kinase inhibitor dasatinib and a key driver in OCCC, ARID1A mutation. Imposing ARID1A deficiency upon a variety of human or mouse cells induced dasatinib sensitivity, both in vitro and in vivo, suggesting that this is a robust synthetic lethal interaction. The sensitivity of $A R I D 1 A$ deficient cells to dasatinib was associated with $\mathrm{G}_{1} / \mathrm{S}$ cell cycle arrest and was dependent upon both p21 and Rb. Using focused siRNA screens and kinase profiling, we showed that $A R I D 1 A$ mutant OCCC tumor cells are addicted to the dasatinib target, YES1. This suggests that dasatinib merits investigation for the treatment of patients with ARID1A mutant OCCC. 


\section{Introduction}

Ovarian clear cell carcinomas (OCCC) comprise between 5 and $25 \%$ of all epithelial ovarian cancers and are often associated with endometriosis (1). Patients with advanced OCCC generally respond poorly to standard platinum based chemotherapy and have a median five-year survival rate of less than $32 \%(2,3)$. Recently, comprehensive DNA sequencing of OCCCs has led to the identification of likely driver mutations in this disease $(4,5)$. The most commonly recurrent genetic event in OCCC is somatic mutation in the tumour suppressor gene ARID1A (AT-Rich Interactive Domain-containing protein 1A) which are present in up to $57 \%$ of patients $(4,5)$. A significant proportion of the tumour-associated somatic ARID1A mutations in OCCC are frame-shift insertion/deletion mutations or nonsense mutations that are predicted to result in premature truncation of the protein $(4,5)$. In addition to being recurrently mutated in OCCC, frame-shift insertion/deletion mutations and nonsense mutations in ARID1A have also been identified in multiple other cancer types such as gastric cancer, renal clear cell cancer and pancreatic tumours (6). Loss of ARID1A expression has also been associated with a shorter progression free survival and resistance to platinum based chemotherapy in OCCC patients (7).

The best-characterised role of the ARID1A protein is as a component of the BAF SWI/SNF chromatin-remodeling complex. The BAF complex plays a key role in modifying the position of nucleosomes on DNA (8-10) and in doing so likely regulates the access of additional proteins to DNA (8). The composition of the BAF complex includes: (i) an ATP dependent DNA helicase, encoded 
by the SMARCA4/BRG1 or SMARCA2/BRM genes; (ii) ARID1A or ARID1B, which each encompass an ARID DNA binding domain; and (iii) a series of additional accessory subunits such as SMARCB1, SMARC1 and 2, SMARCD1-3, SMARCE1, ACTL6A and DFP1-3 (8, 11). ARID1A dysfunction has been associated with a relatively diverse set of phenotypes including defects in cell differentiation, alterations in the control of cell proliferation as well as defects in the repair of DNA $(9,10,12,13)$. However, a precise molecular understanding of how defects in ARID1A lead to each of these phenotypes is not yet clear.

Given the high prevalence of $A R I D 1 A$ mutations in OCCC, identifying drugs that selectively target $A R I D 1 A$ mutant tumour cells could potentially inform the development of new therapeutic approaches to this disease. Recently the methyltransferase, EZH2 and PARP inhibitors have been proposed as therapeutic targets in ARID1A mutant tumours $(14,15)$ and synthetic lethality between ARID1A and ARID1B has also been described (16). Here, we describe a high-throughput functional genomics approach to screen a series of clinically used drugs with the intention of identifying novel ARIDIA synthetic lethal effects (17) that operate in OCCC. By profiling drugs that are either already used in the clinical management of cancer or compounds with targets that are currently in late-stage development, we also aimed to identify ARID1A synthetic lethal approaches that could potentially be rapidly translated in the clinic. 


\section{Materials and Methods}

\section{Reagents and cell lines}

Dasatinib was purchased from Selleck chemicals. Additional drugs and small molecule inhibitors used in this study are listed in Supplementary Table S1. ES2 and TOV21G were obtained from the American Type Tissue Collection. RMG-1, SMOV2, KOC7C, HCH1, OVAS, OVISE, OVMANA, OVTOKO and KK were provided by Dr. Hiroaki Itamochi (Tottori University School of Medicine, Yonago, Japan). Ovarian clear cell lines were grown in RPMI with $10 \%$ FCS. The identity of cell lines was confirmed by STR typing using the StemElite Kit (Promega) in March 2013 and where appropriate, profiles were confirmed using the Children's Oncology Group (COG) Cell Culture and Xenograft Repository bank (http://strdb.cogcell.org) or JCRB cell bank (http://cellbank.nibio.go.jp). ARID1A HCT116 were grown in McCoys media with $10 \%(v / v)$ FCS. Arid1a null and wild-type mouse embryonic stem cells were provided by Dr Zhong Wang (Harvard Medical School, USA) (9) and grown on gelatin coated plates in DMEM with $10 \%(\mathrm{v} / \mathrm{v})$ FCS supplemented with $0.1 \mathrm{mM}$ non essential amino acids, $1 \mathrm{mM}$ sodium pyruvate, $0.1 \mathrm{mM}$ betametcaptothanol and $2000 \mathrm{U} \mathrm{LIF} / \mathrm{ml}$. All cell lines were routinely confirmed as being mycoplasma negative using the MycoAlert Kit (Lonza) throughout experimentation.

\section{High throughput drug screen}

Cell lines were profiled using a customised drug library (Supplementary materials and methods) containing 68 compounds (Supplementary Table S2 
and Supplementary Table S3). Cells were plated at a density of 250 or 500 cells per well. 24-hours later, media containing drug library was added to adherent cells. On day seven, cell viability in each well was estimated using CellTiter-Glo (Promega). Luminescence data was log2 transformed and centred on a per plate basis according to the plate median value. $Z$ prime $>0.3$ and $r^{2}>0.75$ were used to define acceptable screen data. Where appropriate, surviving fractions were calculated relative to DMSO treated wells and this data was used to generate AUC (area under the curve) and $\mathrm{SF}_{50}$ data.

\section{Cell-based assays}

Short-term drug exposure assays were performed in 96 well plates. Cells were plated at a density of $250-500$ cells/ well and drug added at the indicated concentration 24 hours later. Cell viability was estimated after seven days using Cell-Titre Glo (Promega). Surviving fractions (SFs) were calculated relative to DMSO treated wells, and drug sensitivity curves plotted.

Clonogenic assays were performed in triplicate in 6 well plates. OCCC cell lines were plated at a density of 500 cells/well and mouse ES cells were plated at a density of 2000 cells per well on gelatin-coated plates. Media containing dasatinib at the indicated concentrations was replaced every three days. After 14 days, cells were fixed in $10 \%$ trichloroacetic acid and stained with sulforhodamine-B prior to counting.

\section{siRNA experiments}


A 384 well plate arrayed siRNA library targeting 784 genes (Dharmacon) was used (gene list described in Supplementary Table S4) or a bespoke Dharmacon siRNA library of dasatinib targets. Each well either contained a SMART pool (four distinct siRNA species targeting different sequences of the target transcript combined) or a single siRNA species. Additional positive (siPLK1) and negative (siCON1, siCON2 and Allstar (Dharmacon and Qiagen, respectively)) controls were also added to each plate. Cells were reverse transfected using Dharmafect4 (Dharmacon). 48 hours following transfection, media containing with dasatinib or the drug vehicle, DMSO, was added to the plates. After seven days, cell viability in each well was estimated using a CellTitre-Glo assay. Data was processed as described in supplementary materials and methods and as in (18).

\section{Dasatinib Bead Proteomics}

Inhibitor bead proteomics was performed as previously described $(19,20)$. Dasatinib was covalently linked to $\mathrm{ECH}$ sepharose 4B using EDC and then 1 mg of pre-cleared cell lysate was rotated with $50 \square \mathrm{L}$ of dasatinib bead slurry for three hours. Beads were washed twice with cold binding buffer and then an additional three times with binding buffer with reduced $\mathrm{NaCl}$ minus detergent. Proteins were eluted with $6 \mathrm{M}$ urea and trypsinised, desalted and analysed by LC-MS/MS on a Thermo LTQ Orbitrap Velos. Spectra were searched using Protein Prospector and label free quantitation performed with Skyline. Significance of effects was determined using MS Stats (21). 


\section{Protein analysis}

Cells were lysed, electrophoresed and immunoblotted as described previously (22). We used the following antibodies: ARID1A (Bethyl A301-040A [human] and Santa Cruz sc-32761 [mouse]) $\alpha$-Actinin (A5044, Sigma), Actin (sc-1616 Santa Cruz), $\beta$-Tubulin, (2146, cell signaling), YES1 (3201S Cell Signaling) and CDKN1A (2947 Cell Signaling). All secondary antibodies were horseradish peroxidase conjugated. Primary antibodies were used at a concentration of $1: 1000$ and secondary antibodies at a concentration of 1:10000. Protein bands were visualised using enhanced chemiluminescence (ECL, GE Healthcare, UK) and Kodak BioMAX XAR film (Kodak). Alternatively, lysates were probed with the primary antibody and a fluorescent-dye labeled secondary antibody and images were taken using the Odyssey Infrared Imaging system from Licor ${ }^{\circledR}$.

\section{Exome sequencing}

BWA (Burrows-Wheeler Aligner, bio-bwa.sourceforge.net) was used to align short reads to a reference sequence (GRCh37). Duplicate sequence reads (PCR-derived duplicates) were removed from further analysis at this point. Base quality recalibration, realignment around indels, and variant calling were performed using the Genome Analysis Tool Kit (GATK) using the Broad best practice variant detection workflow (www.broadinstitute.org/gatk/guide/bestpractices). Small insertions and deletions detected in the tumour cell lines that were absent in the reference genomes were considered to be candidate somatic mutations. The set of candidate somatic mutations was refined using the following list of heuristic rules: (i) variants called in regions not covered by 
the exome capture probes were excluded; (ii) variants marked as low quality (QUAL below 20) were excluded; (iii) variants with fewer than 10 reads covering the locus in all samples were excluded. Common SNPs (those reported to have a global minor allele frequency of greater than $5 \%$ in any of the 1000 genomes project data sets) were also removed from the main analysis. The remaining variants were annotated using the Ensembl variant effect predictor script (Ensembl v61).

\section{Cell cycle analysis}

Cells were plated at a density of $2 \times 10^{6}$ cells per well of a six well plate and incubated for 24 hours after which dasatinib or $0.1 \%(\mathrm{v} / \mathrm{v})$ DMSO was added to the media and cells cultured for a further 24 hours. After incubation, both adherent and free-floating cells were harvested and then fixed overnight with cold $70 \%(\mathrm{v} / \mathrm{v})$ ethanol. Cells were then treated with RNase A for 30 mins prior to nucleic acid staining with propidium iodide (PI, Sigma). Samples were analyzed on a BD LSR II flow cytometer using BD FACSDiva software (BD Biosciences).

\section{Apoptosis assay}

After 48 hours dasatinib exposure, cells were analysed using the ApoToxGlo ${ }^{\mathrm{TM}}$ Triplex Assay (Promega) as per the manufacturer's instructions.

\section{In vivo efficacy studies}

For the biomarker study, TOV21G cells were used to generate subcutaneous xenografts in nu/nu athymic female mice. Once tumours were established ( $\geq$ $500 \mathrm{~mm}$ diameter), treatment with dasatinib was initiated for 72 hours. 
Dasatinib was either dissolved in $80 \mathrm{mM}$ sodium citrate at $\mathrm{pH} 3$ for administration via oral gavage or dissolved in DMSO for intraperitoneal injections. Three animals received oral dasatinib at a dose of 15,30 or 45 $\mathrm{mg} / \mathrm{kg} / \mathrm{day}$ and three animals IP dasatinib at a dose of 5,10 or $15 \mathrm{mg} / \mathrm{kg} / \mathrm{day}$, the remaining animal served as a control and was not treated. Two hours following the final drug administration animals were culled; tumour and normal tissue (spleen and liver) were then collected. Tissue was then thinly sliced and mixed with RIPPA buffer in a cryotube overnight at $4^{\circ} \mathrm{C}$. Samples were then homogenized and spun in a centrifuge at $15,000 \mathrm{rpm}$ for 15 minutes. The supernatant was then removed and protein quantified using Biorad Protein Assay Reagent (BioRad, Hemel Hempstead, UK).

TOV21G cells were transfected with a luciferin expression lentivirus (LVP433 Amsbio) and maintained in blasticidin selection $(25 \mathrm{ug} / \mathrm{ml})$ for ten days prior to injection into female athymic nu/nu mice. $1.0 \times 10^{6}$ TOV21G cells in $100 \mu \mathrm{l}$ of PBS were injected directly into the peritoneal cavity of $40 \mathrm{nu} / \mathrm{nu}$ athymic female mice. Treatment with dasatinib $(n=20)$ or vehicle $(n=20)$ was initiated 24 hours following tumour injection. Mice received either dasatinib $15 \mathrm{mg} / \mathrm{kg}$ in sodium citrate $(80 \mathrm{mM})$ via oral gavage or vehicle treatment (sodium citrate) daily. Prior to IVIS imaging luciferin $150 \mathrm{mg} / \mathrm{kg}$ (Perkineliner) was injected into the peritoneum. Mice were culled when they exhibited signs of distress or $>20 \%$ weight loss or gain (secondary to ascites). 


\section{Results}

\section{Identification of ARID1A selective drugs using a focused drug screen}

We aimed to identify existing drugs that could selectively target $A R I D 1 A$ mutant OCCC. To do this, we designed a high-throughput drug sensitivity screen (Figure 1A), where we profiled the in vitro sensitivity of a range of tumour cell models of OCCC to 68 drugs many of which are currently used in the treatment of cancer or are in late stage development. To facilitate this, we first characterised a panel of commonly used OCCC tumour cell models according to their ARID1A gene mutation and protein expression status. Using whole exome sequencing and immunoblot analysis, we confirmed the previously documented $(23,24)$ presence of truncating $A R I D 1 A$ mutations and loss of full length ARID1A protein expression in SMOV2, OVISE, TOV21G, OVTOKO, OVMANA, KOC7C, OVAS and HCH1 OCCC cell line models (Supplementary Figures S1A-B, S2 and Supplementary Table S5). We also confirmed the expression of full length ARID1A protein and the absence of ARID1A gene mutations in ES2, KK and RMG-1 models of OCCC (Supplementary Figure S1A-B, S2 and Supplementary Table S5). This characterisation allowed us to define two cohorts of OCCC tumour cell models for further analysis: ARID1A mutant (deficient) (SMOV2, OVISE, TOV21G, OVTOKO, OVMANA, KOC7C, OVAS and $\mathrm{HCH} 1)$ and ARID1A wild type (ES2, KK and RMG-1).

Using these OCCC tumour cell lines, we carried out a series of parallel highthroughput drug sensitivity screens (HTS). As a screening library, we used an in-house curated collection of 68 drugs that were selected on the basis of 
being either already used in the treatment of cancer or being in late stage development (Supplementary Table S2). Each tumour cell line was plated in a 384-well plate format and then 24-hours later, exposed to each drug for a subsequent five days. At the end of this five-day period, we estimated cell viability by the use of a CellTitreGlo (CTG) assay (Figure 1A). Each of the 11 OCCC tumour cell lines was screened in triplicate, with replica drug sensitivity data from each cell line being highly reproducible as shown by Pearson's correlation coefficients between screen replicas of $>0.75$ (Figure 1B and Supplementary Table S6). The dynamic range of each screen was also estimated by calculating $Z$ prime ( $Z$ ') values for data from each 384 well plate used in the screen. To calculate Z' values we compared CTG luminescent readings from DMSO (cell inhibition negative control) exposed cells to CTG luminescence readings from wells where cells were exposed to $5 \mu \mathrm{M}$ puromycin (cell inhibition positive control). Each 384 well plate in the screen delivered a Z' > 0.5 (Figure 1C and Supplementary Table S6), confirming a suitable dynamic range for each plate used in the screen. To maximise the potential for identifying $A R I D 1 A$ synthetic lethal effects, we screened each cell line using four different drug concentrations ( $1 \mathrm{nM}, 10 \mathrm{nM}, 100 \mathrm{nM}$ and $1 \mu \mathrm{M})$ and used this data to generate dose/response survival data for each drug in each cell line model (Supplementary Table S7). This data was then used to calculate area under the curve values (AUC) for each drug in each tumor cell line as well as $\mathrm{SF}_{50}$ (Surviving Fraction 50 - the concentration of drug required to elicit a $50 \%$ inhibition of the cell population) estimates of drug sensitivity (Supplementary Figure S3A, B) of each OCCC tumour cell line. 
By comparing the median AUC values in ARID1A wild type and mutant cohorts, we identified drugs predicted to deliver an ARID1A mutant selective effect. The most profound effect identified in this way was elicited by dasatinib (BMS-354825 - Figure 1D,E). When defining the differential drug sensitivities in ARIDIA mutant vs. wild type cohorts by comparing median AUC values, dasatinib showed a distinct effect, with an AUC of $4 \times 10^{-6}$ in the ARID1A mutant cohort vs. $1 \times 10^{-5}$ in the ARID1A wild type cohort (Figure 1D,E), an effect also observed by the comparison of dasatinib $S_{50}$ data (Figure $1 F, G$ ). Finally, by using an ANOVA calculation to assess the overall difference in surviving fraction across the $1 \mathrm{nM}-1 \mu \mathrm{M}$ dasatinib concentration range used in the screen, we found the ARID1A mutant cohort to have a significantly lower survival that the ARID1A wild type cohort $(p<0.0001$, ANOVA). In addition to dasatinib, we also noted that drugs such as everolimus caused moderate ARIDIA selective effects in the drug screen (Supplementary Table S7, Supplementary Figure S3A). Elements of the PI3K/mTOR signaling cascade have recently been shown to be constitutively active in ARID1A mutant endometrial and OCCC cancers (25-27), suggesting that ARID1A mutant tumours might be addicted to PI3 kinase signaling (28). On the basis of this earlier work and our screen data we assessed these moderate ARID1A selective effects in subsequent validation experiments (Supplementary Figure S4). These confirmed that ARID1A mutant tumour cell line models were modestly more sensitive to drugs such as the everolimus, but these effects were not as profound as those caused by dasatinib (Supplementary Figure S4). 


\section{Dasatinib is a synthetic lethal drug in ARID1A mutant OCCC tumour cell} line models

To validate the ARID1A selectivity of dasatinib identified in the highthroughput screens, we carried out both short-term as well as long-term drug sensitivity experiments in our panel of OCCC tumour cell lines. We found that a relatively short six-day exposure to dasatinib was selective for $A R I D 1 A$ mutant OCCC models (Figure 2A,B, Supplementary Figure S5A-B, two-way ANOVA ARID1A mutant vs. wild-type cohorts $p<0.05)$ as was a longer-term, 15-day drug exposure (Figure 2C,D, Supplementary Figure S5C-D, two-way ANOVA ARID1A mutant vs. wild-type cohorts in Figure 2C $p<0.0001$ ).

Although we found dasatinib to preferentially target the ARID1A mutant cohort of OCCC models, the possibility existed that other genetic variants in the tumour cell line panel might explain the sensitivity to dasatinib. To independently establish whether ARID1A was indeed a determinant of dasatinib sensitivity, we assessed drug sensitivity in three different isogenic model systems in which we engineered either ARID1A depletion or mutation. In the first instance, we exploited the previously validated mouse ES cell model of Arid1a deficiency (Figure 2E and Supplementary Figure S2) generated by Gao and colleagues (9), where both alleles of Arid1a were rendered dysfunctional by gene targeting. We found dasatinib to be selective for the Arid1a null ES cell model, compared to the wild type clone, consistent with the hypothesis that $A R I D 1 A$ is a determinant of dasatinib sensitivity $(p<0.0001$ two-way ANOVA, Figure 2F). We also used gene silencing to suppress ARID1A expression in the two ARID1A wild-type, dasatinib resistant, 
human OCCC models and ARID1A wild-type breast cancer and colorectal cancer cell lines models (Figure 2G, H and Supplementary Figures S2, S5EG). Not only did the siRNA SMARTpool designed to target ARID1A elicit dasatinib sensitivity in the ARID1A wild-type OCCC cell lines but this was also caused by multiple independent ARID1A siRNA species (Figure 2G,H), suggesting that this was unlikely to be an off target effect of the RNA interference reagents used.

In addition to these two systems of ARID1A perturbation, we also generated an isogenic human colorectal tumour cell model (HCT116) in which both copies of the ARID1A gene were inactivated by a p.Q456* truncating mutation. This model was generated by AAV-mediated somatic gene targeting (29). As expected, mutation of both copies of ARID1A (Supplementary Figure S6A) caused loss of full length ARID1A expression (Supplementary Figures S2 and S6B). Using this model $\left(A R I D 1 A^{\mathrm{Q} 456^{*} / \mathrm{Q} 456^{*}}\right)$ and the parental ARID1A wild type clone $\left(A R I D 1 A^{\mathrm{WT} / \mathrm{WT}}\right)$, we carried out a subsequent high-throughput drug screen using a second compound library which included the original screening library with the addition of a number of supplementary drugs (Supplementary Table S3, Supplementary Table S8). Three different concentrations of dasatinib $(1000 \mathrm{nM}, 500 \mathrm{nM}$ and $100 \mathrm{nM})$ were ranked within the ten most profound $A R I D 1 A^{Q 456^{*} / Q 456^{*}}$ selective effects (Supplementary Figure S6C). This finding was confirmed in a subsequent validation assay (Supplementary Figure S6D, two-way ANOVA $p<0.001$ ARID1A ${ }^{Q 456^{*} / Q 456^{*}}$ cells compared to the parental ARID1A ${ }^{\mathrm{WT} / \mathrm{WT}}$ model). 
Having confirmed the validity of the ARID1A/dasatinib synthetic lethality, we assessed whether this effect could be explained by hyperactivity and/or addiction to dasatinib targets. In the first instance, we used an unbiased activity-based proteomics approach to estimate the relative activity of all dasatinib-binding kinases in a subset of our OCCC panel. This "inhibitor bead" approach is based upon the use of an ATP-competitive kinase inhibitor (in this case dasatinib) covalently linked to sepharose. As the ATP-binding avidity of protein kinases is increased by the allosteric changes that follow kinase activation, the relative amount of particular kinases bound to sepharosedasatinib beads (measured by mass spectrometry) can be used to estimate kinase activity (Figure 3A) $(19,30,31)$. Using this approach, we profiled OVISE, KK, RMG1 and TOV21G cells and found that five dasatinib targets (EPHA2, MAP4K5, ABL2, YES1 and ABL1) were significantly $(p<0.01)$ enriched in the ARID1A mutant cell lines compared to the wild type cells (Figure 3B, Supplementary Tables S9 and S10).

Although this data suggested that ARID1A mutant tumour cells exhibited enhanced activity of a number of dasatinib targets, it did not formally confirm addiction to EPHA2, MAP4K5, ABL2, YES1 or ABL1. Therefore, in parallel with this proteomic assessment we also used a genetic approach and assessed the addiction of 10 OCCC tumour cell lines (three ARID1A wild type and seven ARID1A mutant models) to dasatinib targets (Figure $3 C$ ). We selected 14 dasatinib targets that have dasatinib dissociation constants $\left(k_{d}\right)$ of $<1 \mathrm{nM}$ (32) and are inhibited at clinically relevant concentrations (33) (Supplementary Table S11) and profiled each tumour cell model with this 
library. Using this data, we were able to calculate median NPI values for both the ARID1A mutant OCCC cell line cohort (SMOV2, OVISE, TOV21G, OVMANA, KOC7C, OVAS and $\mathrm{HCH} 1$ ) as well as the ARID1A wild type cohort (KK, ES2 and RMG-1) (Figure 3C). By comparing the cell inhibitory effects caused by each siRNA in ARID1A wild-type and mutant cohorts, we identified likely ARID1A synthetic lethal effects (Supplementary Table S11, Figure 3D, Supplementary Figure S7A). We noted that the most consistent ARID1A selective effect (i.e. where the ARID1A selective effect was observed with multiple different siRNAs) was caused by siRNA designed to target YES1 (Figure 3D). Three of the YES1 individual siRNA species and the YES1 siRNA SMARTpool ranked within the ten most profound ARID1A selective effects, as defined by the median difference in NPI (Figure 3E,F, Supplementary Figures S2, S7B and Supplementary Table S11). We also found that the YES1 siRNA SMARTpool and individual YES1 siRNAs selectively targeted the $A R I D 1 A^{Q 456^{*} / Q 456^{*}}$ HCT116 clone' as opposed to the $A R I D 1 A^{\mathrm{WT} / \mathrm{WT}}$ parental clone (Supplementary Figure S7C), suggesting that ARID1A mutation might indeed cause dependency upon YES1, an observation that could explain the sensitivity of $A R I D 1 A$ mutant tumour cells to dasatinib. We confirmed that YES1 activation was reduced at relevant dasatinib concentrations (Supplementary Figures S2, S7D and S7E). However, the addiction to YES1 in ARDIIA mutant cell lines could not be explained by baseline differences in expression levels between the ARID1A wild-type and mutant cohorts (Supplementary Figures S2 and E-F). 


\section{Dasatinib sensitivity in ARID1A mutant OCCC is p21 and RB dependent and is characterised by an apoptotic response}

One of the known phenotypic effects of dasatinib exposure in tumour cell lines is the induction of $G_{1}$ cell cycle arrest followed by cell apoptosis (34-39). To understand whether a similar effect might explain the ARID1A/dasatinib synthetic lethality, we assessed cell cycle progression and the extent of apoptosis in two ARID1A mutant and two wild-type OCCC models. This was carried out using propidium iodide and annexin $\mathrm{V}$ staining, followed by Fluorescent Activated Cell Scanning (FACS). We observed a modest but significant increase in dasatinib induced $\mathrm{G}_{1}$ arrest in the ARID1A mutant models, $\mathrm{HCH} 1$ and OVISE compared to wild type models, ES2 and KK (Figure 4A, $p<0.05$ in each wild-type vs. mutant comparison, Student's t-test). There was however a more profound apoptotic response to dasatinib in the ARID1A mutant models suggesting that although a cell cycle response could form part of the dasatinib effect, apoptosis might also play a role in the cell growth inhibition observed ( $p$-values for ARID1A mutant cells [OVISE and TOV21G] cells versus ARID1A wild-type [ES2 and KK] $p<0.05$ Student's t-test, Figure 4B and Supplementary Figure S8).

To further investigate what the key determinants of the ARID1A/dasatinib synthetic lethality might be, we used a synthetic rescue genetic screen to identify genes which when inactivated drove dasatinib resistance in ARID1A null OCCC models. To do this, we transfected the ARID1A mutant (p.542fs), dasatinib sensitive $\left(\mathrm{SF}_{50}=23 \mathrm{nM}\right)$ OVISE OCCC model with a siRNA library targeting 784 genes, predominantly protein kinase coding genes, and 
assessed whether we could induce dasatinib resistance. OVISE cells were reverse transfected in 384-well plates containing the siRNA library. In total, nine replica transfections were performed. Forty-eight hours after siRNA transfection cells were exposed to either $25 \mathrm{nM}$ dasatinib (three replicates), $120 \mathrm{nM}$ dasatinib (three replicates) or the drug vehicle DMSO (three replicates). Cells were then continuously exposed to drug for five days after which cell viability was estimated by use of a CellTitreGlo (CTG) assay. In cells transfected with a control non-targeting siRNA (siCON), exposure to 25 $\mathrm{nM}$ dasatinib resulted in a Surviving Fraction of $50 \%\left(\mathrm{SF}_{50}\right)$, whereas exposure to $120 \mathrm{nM}$ dasatinib resulted in a SF on $16 \%\left(\mathrm{SF}_{16}\right)$. The effect of each siRNA on dasatinib resistance was then estimated by calculating drug effect (DE) Z scores ((18) and see Materials and Methods) with positive DE Z scores representing resistance causing effects. For each gene, the $D E Z$ scores at 25 and $125 \mathrm{nM}$ dasatinib were calculated and rank ordered (Figure 5A and Supplementary Table S12). By calculating the average DE Z scores from both $\mathrm{SF}_{50}$ and $\mathrm{SF}_{16}$ screens, we identified those siRNAs that elicited the most robust dasatinib resistance-causing effects.

Using this relatively unbiased approach, we found the most profound dasatinib resistance causing effects in both $\mathrm{SF}_{50}$ and $\mathrm{SF}_{16}$ screens to be caused by siRNAs targeting the key $\mathrm{G}_{1} / \mathrm{S}$ cell cycle regulators $C D K N 1 A(p 21)$ and $R B 1$ (DE $Z$ scores of 4.41 and 4.96 respectively, Figure 5A). Dasatinib has been shown to increase the expression of $\mathrm{p} 21$, the protein encoded by $C D K N 1 A$, as well as enhancing the protein expression of another cyclin dependent kinase inhibitor, p27 $(34,36,38-41)$. Considering this, as well as 
the results from the synthetic rescue screen described above, we assessed whether the dasatinib/ARID1A synthetic lethality could be reversed by inactivation of $C D K N 1 A$ or the downstream mediator, RB1. We found that an siRNA pool designed to target CDKN1A reversed the dasatinib/ARID1A synthetic lethality in the ARID1A mutant OCCC cell line OVISE but had negligible effects in an ARID1A wild-type model ES2 (Figure 5B and Supplementary Figure S9A). In order to confirm that the dasatinib resistance observed was due to CDKN1A silencing and not an off-target effect, three individual siRNA species and the SMARTpool were transfected into two ARID1A mutant OCCC cell lines and the ARID1A isogenic clones. Transfection of each individual siRNA and the SMARTpool led to dasatinib resistance in both of the cell lines examined suggesting an on-target effect (Figure $5 \mathrm{C}$ ) and western blot analysis confirmed CDKN1A silencing (Figure 5D and Supplementary Figure S2). We also found that silencing of RB1, a key modulator of the $G_{1} / S$ checkpoint also caused dasatinib resistance in multiple ARID1A mutant OCC cell lines and the isogenic HCT116 ARID1A Q456*/Q456* clone (Supplementary Figures S2 and S9B-E), supporting the hypothesis that in $A R I D 1 A$ null tumour cells, the inhibitory effect of dasatinib is mediated via the activity of CDKN1A and RB1.

\section{Dasatinib is selective for ARID1A mutant OCC tumours in vivo}

We generated an in vivo model of ARID1A mutant OCCC by subcutaneously xenografting the TOV21G ARID1A mutant OCCC cell line (ARID1A p.548fs/p.756fs) into immunocompromised recipient mice. This led to the growth of very aggressive, highly proliferative, subcutaneous tumours. This 
approach allowed us to assess the effect of dasatinib on these tumours. Published reports on the use of dasatinib in mice vary greatly in terms of the route of administration (oral gavage or intraperitoneal (IP) injections) as well as in the dasatinib dose used (42-44). Therefore, to identify a dose and route of administration that would minimize the impact of deleterious side effects whilst still delivering an effective dose, we first conducted a biomarker study. Subcutaneous TOV21G tumours were established in nu/nu athymic mice and animals were treated with dasatinib using either oral gavage or intraperitoneal (IP) routes of administration at a range of concentrations (oral administration of 15,30 or $45 \mathrm{mg} / \mathrm{kg}$ per day or IP administration of 5,10 or 15 $\mathrm{mg} / \mathrm{kg}$ per day). By estimating the extent of SRC phosphorylation as a biomarker of dasatinib activity, we were able to predict whether dasatinib elicited a mechanistic effect in both tumour and normal tissue (Supplementary Figure S10). At each dasatinib dose tested and using ether oral or IP administration, SRC phosphorylation was completely abolished in both tumour and normal tissue (Supplementary Figure S10) and was well tolerated in each case. We therefore selected the lowest dose of dasatinib $(15 \mathrm{mg} / \mathrm{kg}$ ) for subsequent studies and administered this via an oral route.

Based on this data, we assessed the in vivo anti-tumour efficacy of dasatinib. To do this, we first labeled ARID1A mutant TOV21G cells with a luciferaseexpressing construct so that we could later visualize the extent of tumour burden in live animals by the use of an In Vivo Imaging System (IVIS, PerkinElmer). These labeled cells were then xenografted into recipient mice where they generated widespread miliary (disseminated) peritoneal disease 
and ascites formation, reminiscent of the clinical scenario in OCCC (Figure $6 \mathrm{~A})$. Treatment of these mice with dasatinib led to a significant reduction in TOV21G-related luminescence (Figure 6A-B $p<0.01$ two-way ANOVA), suggesting a therapeutic response. This effect was also reflected in an increase in overall survival in the dasatinib treated cohort of mice $(p=0.004$ Mantel Cox test).

\section{Discussion}

Ovarian cancer is the most lethal gynaecological malignancy. The OCCC subtype presents a particular clinical management challenge as it is invariably characterised by resistance to standard chemotherapy as well as a poor prognosis (1). In the work presented here, we have attempted to understand drug sensitivities associated with $A R I D 1 A$ mutation in OCCC by using a functional genomics approach. This has led us to the identification of dasatinib as a candidate synthetic lethal drug in ARID1A mutant OCCC. Dasatinib is not only selective for ARID1A mutant OCCC models, but experimental induction of ARID1A deficiency drives dasatinib sensitivity in ARID1A wild type OCCC models as well as in isogenic cell systems (both mouse and human) where ARID1A has been rendered dysfunctional by gene targeting. The selectivity of dasatinib in this context appears to be characterized by a cell cycle arrest/apoptotic phenotype, which can be reversed with silencing of either CDKN1A or RB1.

$A R I D 1 A$ has previously been demonstrated to act as a negative regulator of the cell cycle $(10,24,45)$. In part this effect has been ascribed to an 
impairment of p21 mediated cell cycle control in ARID1A null cells (24). Taking this into account, along with the data presented here, and the known role of dasatinib in p21 induction $(34,36,46)$, one possibility is that dasatinib targets ARID1A mutant tumour cells because it reverses p21 dysfunction caused by ARID1A deficiency. The work presented here suggests that the dasatinib sensitivity of ARID1A null models is somewhat dependent upon p21 and RB1, observations that are consistent with this hypothesis. It is possible that the sensitivity to dasatinib might be related to a dependency upon the dasatinib target YES1 that we have observed, although we cannot discount the possibility that other dasatinib targets might also be involved or that combinations of dasatinib targets might play a role in the phenotype we observe. We do note that an analysis of microarray-based mRNA profiles from the ARID1A wild type and mutant tumour cell lines described in this report and publically available transcriptomic profiles of ARID1A mutant OCCC did not identify a canonical signaling process that was distinct between wild type and mutant cohorts (RM, CJL, AA, unpublished observations). Although the correlation between ARID1A genotype and dasatinib sensitivity are striking, being present in not only in the panel of OCCC models but also in isogenic systems, we noted that one of the ARID1A mutant OCCC cell lines, KOC7C, was relatively resistant to dasatinib but addicted to YES1. It is possible that whilst the ARID1A defect in KOC7C is sufficient to drive YES1 addiction, other genetic or epigenetic factors in this cell line modulate the ARID1A/dasatinib synthetic lethality. 
Our assessment of dasatinib efficacy suggests that there are promising signs that dasatinib can inhibit $A R I D 1 A$ mutant tumours in vivo, although it is clear that further work is required to optimise how dasatinib might be used to elicit a profound, long-lasting anti-tumour response in women with ovarian clear cell carcinomas. For example, using additional OCCC cell lines (both ARID1A wild type and mutant) as established orthotopic tumours (as opposed to nonestablished tumours shown in Figure 6) would extend the observations made using the TOV21G model. It also seems reasonable to suggest that combination therapy approaches involving dasatinib might be used to maximise the therapeutic window as well as minimising the impact of signalling feedback loops that might impair the therapeutic effect of dasatinib. In this regard, one clear objective for future in vivo assessment will be to assess the possibility that the therapeutic window caused by dasatinib could be enhanced by combining this kinase inhibitor with other proposed ARID1A synthetic lethal drugs such as EZH2 and PARP inhibitors $(14,15)$. It might also be pertinent to assess the impact of tumour heterogeneity on the dasatinib therapeutic response. In this case subsequent studies assessing the effect of dasatinib in mice bearing $A R I D 1 A$ mutant human patient derived xenografts (PDXs) might be appropriate, given that PDX material has the capacity to reflect the molecular heterogeneity of human tumours.

Dasatinib is already licensed for use for the treatment of chronic myelogenous leukemia (CML) and Philadelphia chromosome positive acute lymphoblastic leukemia $(A M L)(47,48)$. Several clinical trials have also been conducted, or are at least underway, in patients with solid tumours. In these solid tumour 
clinical trials, dasatinib is being assessed either as a single agent therapy or is being assessed when used in combination with cytotoxic or other targeted therapies (49). However, to date there has been limited success in using dasatinib in the treatment of ovarian cancer patients. When dasatinib was assessed as a single agent therapy in patients with relapsed epithelial ovarian cancer, minimal anti-tumour activity was observed, although we note there were only two patients with OCCC in this study, neither of which had known ARID1A status $(50,51)$. In a recent phase one-clinical trial using dasatinib in combination with standard cytotoxic chemotherapy (carboplatin and paclitaxel), the drug combination regime could be delivered safely and some evidence of clinical efficacy was achieved $(50,51)$. Of course, neither of these trials were designed to test the hypothesis that ARID1A mutant OCCC might respond to dasatinib therapy and based upon the work we describe in this manuscript, we believe that testing this hypothesis is warranted.

\section{Acknowledgments}

We thank Prof. Stan Kaye (ICR and Royal Marsden Hospital, London, UK) for useful discussions. We also thank Dr Zhong Wang (Harvard Medical School, USA) for providing Arid1a null and wild-type mouse embryonic stem cells. This work was supported by a Cancer Research UK Programme Grant (C347/A8363) as well as funding from Breast Cancer Now and the European Union as part of the EUROCAN FP7 Programme (HEALTH-FP7-2010260791). 


\section{References}

1. Anglesio MS, Carey MS, Kobel M, Mackay H, Huntsman DG. Clear cell carcinoma of the ovary: a report from the first Ovarian Clear Cell Symposium, June 24th, 2010. Gynecologic oncology. 2011;121:407-15.

2. Chan JK, Teoh D, Hu JM, Shin JY, Osann K, Kapp DS. Do clear cell ovarian carcinomas have poorer prognosis compared to other epithelial cell types? A study of 1411 clear cell ovarian cancers. Gynecologic oncology. 2008;109:370-6.

3. Pectasides D, Fountzilas G, Aravantinos G, Kalofonos C, Efstathiou H, Farmakis D, et al. Advanced stage clear-cell epithelial ovarian cancer: the Hellenic Cooperative Oncology Group experience. Gynecologic oncology. 2006;102:285-91.

4. Jones S, Wang TL, Shih le M, Mao TL, Nakayama K, Roden R, et al. Frequent mutations of chromatin remodeling gene ARID1A in ovarian clear cell carcinoma. Science. 2010;330:228-31.

5. Wiegand KC, Shah SP, Al-Agha OM, Zhao Y, Tse K, Zeng T, et al. ARID1A mutations in endometriosis-associated ovarian carcinomas. The New England journal of medicine. 2010;363:1532-43.

6. Gao J, Aksoy BA, Dogrusoz U, Dresdner G, Gross B, Sumer SO, et al. Integrative analysis of complex cancer genomics and clinical profiles using the cBioPortal. Science signaling. 2013;6:pl1.

7. Katagiri A, Nakayama K, Rahman MT, Rahman M, Katagiri H, Nakayama N, et al. Loss of ARID1A expression is related to shorter progression-free survival and chemoresistance in ovarian clear cell carcinoma. Modern pathology : an official journal of the United States and Canadian Academy of Pathology, Inc. 2012;25:282-8.

8. Wilson BG, Roberts CW. SWI/SNF nucleosome remodellers and cancer. Nature reviews Cancer. 2011;11:481-92.

9. Gao X, Tate P, Hu P, Tjian R, Skarnes WC, Wang Z. ES cell pluripotency and germ-layer formation require the SWI/SNF chromatin remodeling component BAF250a. Proceedings of the National Academy of Sciences of the United States of America. 2008;105:6656-61.

10. Nagl NG, Jr., Patsialou A, Haines DS, Dallas PB, Beck GR, Jr., Moran E. The p270 (ARID1A/SMARCF1) subunit of mammalian SWI/SNF-related complexes is essential for normal cell cycle arrest. Cancer research. 2005;65:9236-44.

11. Weissman B, Knudsen KE. Hijacking the chromatin remodeling machinery: impact of SWI/SNF perturbations in cancer. Cancer research. 2009;69:8223-30.

12. Inoue H, Furukawa T, Giannakopoulos S, Zhou S, King DS, Tanese N. Largest subunits of the human SWI/SNF chromatin-remodeling complex promote transcriptional activation by steroid hormone receptors. The Journal of biological chemistry. 2002;277:41674-85.

13. Lemon B, Inouye C, King DS, Tjian R. Selectivity of chromatinremodelling cofactors for ligand-activated transcription. Nature. 2001;414:9248.

14. Bitler BG, Aird KM, Garipov A, Li H, Amatangelo M, Kossenkov AV, et al. Synthetic lethality by targeting EZH2 methyltransferase activity in ARID1Amutated cancers. Nature medicine. 2015;21:231-8. 
15. Shen J, Peng Y, Wei L, Zhang W, Yang L, Lan L, et al. ARID1A Deficiency Impairs the DNA Damage Checkpoint and Sensitizes Cells to PARP Inhibitors. Cancer discovery. 2015;5:752-67.

16. Helming KC, Wang X, Wilson BG, Vazquez F, Haswell JR, Manchester $\mathrm{HE}$, et al. ARID1B is a specific vulnerability in ARID1A-mutant cancers. Nature medicine. 2014.

17. Ashworth A, Lord CJ, Reis-Filho JS. Genetic interactions in cancer progression and treatment. Cell. 2011;145:30-8.

18. Lord CJ, Martin SA, Ashworth A. RNA interference screening demystified. Journal of clinical pathology. 2009;62:195-200.

19. Martins MM, Zhou AY, Corella A, Horiuchi D, Yau C, Rakshandehroo T, et al. Linking tumor mutations to drug responses via a quantitative chemicalgenetic interaction map. Cancer discovery. 2015;5:154-67.

20. Sos ML, Levin RS, Gordan JD, Oses-Prieto JA, Webber JT, Salt M, et al. Oncogene mimicry as a mechanism of primary resistance to BRAF inhibitors. Cell reports. 2014;8:1037-48.

21. Choi M, Chang CY, Clough T, Broudy D, Killeen T, MacLean B, et al. MSstats: an $R$ package for statistical analysis of quantitative mass spectrometry-based proteomic experiments. Bioinformatics. 2014;30:2524-6.

22. Brough R, Frankum JR, Sims D, Mackay A, Mendes-Pereira AM, Bajrami I, et al. Functional Viability Profiles of Breast Cancer. Cancer discovery. 2011;1:260-73.

23. Anglesio MS, Wiegand KC, Melnyk N, Chow C, Salamanca C, Prentice LM, et al. Type-specific cell line models for type-specific ovarian cancer research. PloS one. 2013;8:e72162.

24. Guan B, Wang TL, Shih le M. ARID1A, a factor that promotes formation of SWI/SNF-mediated chromatin remodeling, is a tumor suppressor in gynecologic cancers. Cancer research. 2011;71:6718-27.

25. Huang HN, Lin MC, Huang WC, Chiang YC, Kuo KT. Loss of ARID1A expression and its relationship with PI3K-Akt pathway alterations and ZNF217 amplification in ovarian clear cell carcinoma. Modern pathology : an official journal of the United States and Canadian Academy of Pathology, Inc. 2013.

26. Liang H, Cheung LW, Li J, Ju Z, Yu S, Stemke-Hale K, et al. Wholeexome sequencing combined with functional genomics reveals novel candidate driver cancer genes in endometrial cancer. Genome research. 2012;22:2120-9.

27. Wiegand KC, Hennessy BT, Leung S, Wang Y, Ju Z, McGahren M, et al. A functional proteogenomic analysis of endometrioid and clear cell carcinomas using reverse phase protein array and mutation analysis: protein expression is histotype-specific and loss of ARID1A/BAF250a is associated with AKT phosphorylation. BMC cancer. 2014;14:120.

28. Samartzis EP, Gutsche K, Dedes KJ, Fink D, Stucki M, Imesch P. Loss of ARID1A expression sensitizes cancer cells to PI3K- and AKT-inhibition. Oncotarget. 2014;5:5295-303.

29. Khan IF, Hirata RK, Russell DW. AAV-mediated gene targeting methods for human cells. Nature protocols. 2011;6:482-501.

30. Wodicka LM, Ciceri P, Davis MI, Hunt JP, Floyd M, Salerno S, et al. Activation state-dependent binding of small molecule kinase inhibitors: structural insights from biochemistry. Chemistry \& biology. 2010;17:1241-9. 
31. Bantscheff $M$, Eberhard D, Abraham $Y$, Bastuck S, Boesche $M$, Hobson S, et al. Quantitative chemical proteomics reveals mechanisms of action of clinical ABL kinase inhibitors. Nature biotechnology. 2007;25:103544.

32. Karaman MW, Herrgard S, Treiber DK, Gallant $P$, Atteridge CE, Campbell BT, et al. A quantitative analysis of kinase inhibitor selectivity. Nature biotechnology. 2008;26:127-32.

33. Kitagawa D, Yokota K, Gouda M, Narumi Y, Ohmoto H, Nishiwaki E, et al. Activity-based kinase profiling of approved tyrosine kinase inhibitors. Genes to cells : devoted to molecular \& cellular mechanisms. 2013;18:110-22.

34. Guerrouahen BS, Futami M, Vaklavas C, Kanerva J, Whichard ZL, Nwawka K, et al. Dasatinib inhibits the growth of molecularly heterogeneous myeloid leukemias. Clinical cancer research : an official journal of the American Association for Cancer Research. 2010;16:1149-58.

35. Inge LJ, Fowler AJ, Paquette KM, Richer AL, Tran N, Bremner RM. Dasatinib, a small molecule inhibitor of the Src kinase, reduces the growth and activates apoptosis in pre-neoplastic Barrett's esophagus cell lines: evidence for a noninvasive treatment of high-grade dysplasia. The Journal of thoracic and cardiovascular surgery. 2013;145:531-8.

36. Johnson FM, Saigal B, Talpaz M, Donato NJ. Dasatinib (BMS-354825) tyrosine kinase inhibitor suppresses invasion and induces cell cycle arrest and apoptosis of head and neck squamous cell carcinoma and non-small cell lung cancer cells. Clinical cancer research : an official journal of the American Association for Cancer Research. 2005;11:6924-32.

37. Michels S, Trautmann M, Sievers E, Kindler D, Huss S, Renner M, et al. SRC signaling is crucial in the growth of synovial sarcoma cells. Cancer research. 2013;73:2518-28.

38. Song Y, Sun X, Bai WL, Ji WY. Antitumor effects of Dasatinib on laryngeal squamous cell carcinoma in vivo and in vitro. European archives of oto-rhino-laryngology : official journal of the European Federation of OtoRhino-Laryngological Societies. 2013;270:1397-404.

39. Le XF, Mao W, He G, Claret FX, Xia W, Ahmed AA, et al. The role of p27(Kip1) in dasatinib-enhanced paclitaxel cytotoxicity in human ovarian cancer cells. Journal of the National Cancer Institute. 2011;103:1403-22.

40. Le XF, Mao W, Lu Z, Carter BZ, Bast RC, Jr. Dasatinib induces autophagic cell death in human ovarian cancer. Cancer. 2010;116:4980-90.

41. Song L, Morris M, Bagui T, Lee FY, Jove R, Haura EB. Dasatinib (BMS-354825) selectively induces apoptosis in lung cancer cells dependent on epidermal growth factor receptor signaling for survival. Cancer research. 2006;66:5542-8.

42. Chan D, Tyner JW, Chng WJ, Bi C, Okamoto R, Said J, et al. Effect of dasatinib against thyroid cancer cell lines in vitro and a xenograft model in vivo. Oncology letters. 2012;3:807-15.

43. Garcia-Gomez A, Ocio EM, Crusoe E, Santamaria C, HernandezCampo P, Blanco JF, et al. Dasatinib as a bone-modifying agent: anabolic and anti-resorptive effects. PloS one. 2012;7:e34914.

44. Nagathihalli NS, Merchant NB. Src-mediated regulation of E-cadherin and EMT in pancreatic cancer. Frontiers in bioscience. 2012;17:2059-69.

45. Flores-Alcantar A, Gonzalez-Sandoval A, Escalante-Alcalde D, Lomeli $H$. Dynamics of expression of ARID1A and ARID1B subunits in mouse 
embryos and in cells during the cell cycle. Cell and tissue research. 2011;345:137-48.

46. Ling C, Chen G, Chen G, Zhang Z, Cao B, Han K, et al. A deuterated analog of dasatinib disrupts cell cycle progression and displays anti-non-small cell lung cancer activity in vitro and in vivo. International journal of cancer Journal international du cancer. 2012;131:2411-9.

47. Hochhaus $A$, Kantarjian $H$. The development of dasatinib as a treatment for chronic myeloid leukemia $(\mathrm{CML})$ : from initial studies to application in newly diagnosed patients. Journal of cancer research and clinical oncology. 2013;139:1971-84.

48. Liu-Dumlao T, Kantarjian H, Thomas DA, O'Brien S, Ravandi F. Philadelphia-positive acute lymphoblastic leukemia: current treatment options. Current oncology reports. 2012;14:387-94.

49. Puls LN, Eadens M, Messersmith W. Current status of SRC inhibitors in solid tumor malignancies. The oncologist. 2011;16:566-78.

50. Schilder RJ, Brady WE, Lankes HA, Fiorica JV, Shahin MS, Zhou XC, et al. Phase II evaluation of dasatinib in the treatment of recurrent or persistent epithelial ovarian or primary peritoneal carcinoma: a Gynecologic Oncology Group study. Gynecologic oncology. 2012;127:70-4.

51. Secord AA, Teoh DK, Barry WT, Yu M, Broadwater G, Havrilesky LJ, et al. A phase I trial of dasatinib, an SRC-family kinase inhibitor, in combination with paclitaxel and carboplatin in patients with advanced or recurrent ovarian cancer. Clinical cancer research : an official journal of the American Association for Cancer Research. 2012;18:5489-98. 


\section{Figure Legends}

Figure 1. Identification of ARID1A selective drugs using a focused drug screen (A) Focused drug high-throughput screen (HTS) schematic. Eight ARID1A mutant and three ARID1A wild type OCCC cell lines were plated in triplicate 384 well plates and twenty four hours later, media containing compound library was added to the 384 well plates. Cells were continuously cultured for a subsequent five days after which cell viability was estimated using a luminescence assay (CellTitre-Glo®, Promega). After processing data (see Materials and Methods) the screen quality control was assessed by examining the correlation between data from replica screens (example shown in B) and Z prime statistics for each plate and each screen replica (example shown in C). (B) Example, scatter plot of drug sensitivity data from replica ES2 cell line screens. (C) Example Z prime values for replica ES2 screens (R1= replica 1$)$. The distribution curve on the left represents the data from positive control (siPLK1) and the curve on the right from negative controls (siCON). (D,E) Waterfall and box-whisker plots of dasatinib AUC data from the high-throughput screen. Median $A R I D 1 A$ mutant $A U C=4 \times 10^{6}$ and wildtype $=$ AUC $1 \times 10^{5}(F, G)$ Waterfall $(F)$ and box-whisker $(G)$ plots of dasatinib $\mathrm{SF}_{50}$ data from the high-throughput screen. Median $A R I D 1 A$ mutant $\mathrm{SF}_{50}=$ $271 \mathrm{nM}$ as opposed to wild-type $\mathrm{SF}_{50}=707 \mathrm{nM}$.

Figure 2. Dasatinib is a synthetic lethal drug in ARID1A mutant OCCC tumour cell line models. (A) Median dasatinib surviving fraction (SF) doseresponse curves in ARID1A mutant and wild-type cohorts. Cells were plated in 96 well plates and exposed to dasatinib for five days after which SF were 
calculated. Data in (A) represents median of six replicates per cell line. Error bars represent SEM. Dasatinib response in ARID1A mutant vs. wild-type cohort, two-way ANOVA $p<0.0001$. (B) Waterfall plot showing the individual dasatinib $\mathrm{SF}_{50}$ for each OCCC cell line (nM). (C) Clonogenic (15 day exposure) dasatinib response in ARID1A mutant vs. wild-type cohort, two-way ANOVA $p<0.0001$. (D) Representative clonogenic assay images from two ARID1A wild-type and two ARID1A mutant cell lines exposed to dasatinib (E) Western blot analysis of Arid1a expression from whole cell lysates in Arid1a isogenic mouse ES cells. Tubulin is included as a loading control. (F) Dasatinib dose response survival data from clonogenic experiments in isogenic ES cells. Cells were plated on gelatin-coated plates and exposed to dasatinib for 14 days. Error bars represent SEM from six replicas per cell line. SF for the Arid1a null versus Arid1a wild-type mouse ES cells, two-way ANOVA $p<0.001$. (G) Dasatinib dose response survival data from the ARID1A wild-type cells (ES2) transfected with siRNA targeting ARID1A. 48 hours following transfection cells were plated into 96 well plates and exposed to dasatinib for five days. Error bars represent SEM from six replica experiments. (H) Western blot of ARID1A expression from whole cell lysates (ES2) transfected as described in (G). Tubulin is included as a loading control.

\section{Figure 3. ARID1A mutant cell line models and YES1 addiction}

(A) Schematic of inhibitor bead approach: active kinases are preferentially purified by dasatinib coupled to sepharose and analysed by LC/MS/MS. Label-free quantification with MS1 filtering is used to determine relative representation of each peptide. (B) Scatter plot of dasatinib bound kinase 
abundance from pooled biological replicates (two each) of ARID1A wild-type (KK, RMG-1) and mutant (OVISE, TOV21G) tumour cell lines. Axes represent median centered abundance; Statistically significant ratios between ARID1A mutant and wild type cell lines (adjusted $p<0.05$ ) are shown in red. (C) Dasatinib target siRNA screen overview. Ten OCCC cell lines were included in the screen. Each cell line was reverse transfected with either a SMARTpool of four siRNAs designed or individual siRNA species (four per gene) in a 384well plate. The siRNA library targeted known targets of dasatinib. After siRNA transfection, cells were cultured for a subsequent five days at which point cell viability was estimated by the use of Cell Titre Glo Reagent. To estimate the extent by which each siRNA caused tumour cell inhibition, Normalised Percent Inhibition (NPI) values for each siRNA were calculated and median NPI values between ARID1A wild type and mutant cohorts compared. (D) Heatmap showing Normalised Percent Inhibition (NPI) data from the dasatinib target siRNA screen. Individual siRNAs are ranked according to difference in NPI between ARID1A mutant and wild-type cohorts. The ten most profound ARID1A selective are highlighted. (E) Bar chart plot of YES1 NPI values from the screen. Median NPI values from each cohort are shown and error bars represent SEM. In each case the NPI in the ARID1A mutant cell line was significantly less than in the wild-type model, $p<0.05$ Student's test. (F) Western blot analysis demonstrating YES1 silencing caused by each individual siRNA species in the OVISE cell line. The OVISE cell line was transfected with either control siRNA, individual YES1 siRNAs, YES1 SMARTpool or mock transfected. 48 hours following transfection whole cell 
lysates were collected. Western blot was probed for YES1 and ACTIN was included as a loading control.

Figure 4. Dasatinib sensitivity in ARID1A mutant OCCC is characterised by $\mathbf{G 1}$ arrest and an apoptotic response. (A) Column chart illustrating the proportion of cells in different phases of the cell cycle when exposed to dasatinib. Exposure to dasatinib led to a significant increase in the proportion of cells in the $\mathrm{G} 1$ phase of the cell cycle in the ARID1A mutant cell lines $\mathrm{HCH} 1$ and OVISE but not the ARID1A wild-type cell lines ES2 and KK. Cells were exposed to $50 \mathrm{nM}$ dasatinib for 24 hours and fixed in $70 \%$ ethanol prior to propidium iodide staining and FACS analysis. Error bars represent \pm SD from $\mathrm{n}=3$ independent experiments. $S$ phase fraction in $A R I D 1 A$ mutant tumour cells is significantly reduced by dasatinib exposure (Student's t-test $\mathrm{p}<0.05$, for both OVISE and $\mathrm{HCH} 1$ ). (B) Bar chart illustrating caspase 3/7 activity in response to dasatinib. Cells were treated as in (A) and caspase 3/7 activity assessed using the ApoTox Glo triplex assay (Promega). p-values for ARID1A mutant cells (OVISE and TOV21G) cells versus ARID1A wild-type $\left(E S 2\right.$ and KK) ${ }^{* * *} p<0.001,{ }^{* *} p<0.01$ Student's t-test.

Figure 5. Dasatinib sensitivity in ARID1A mutant OCCC is dependent upon G1/S checkpoint effectors. (A) Drug effect (DE) Z score distribution plots from two independent dasatinib resistance siRNA screens in OVISE tumour cells. Data from $\mathrm{SF}_{50}$ (left) and $\mathrm{SF}_{16}$ (right) dasatinib resistant screens are shown. CDKN1A and RB1 siRNA effects are highlighted, driving dasatinib resistance in each screen. (B) Dasatinib dose response curves for the 
ARID1A mutant OCCC cell line, OVISE and the ARID1A wild-type cell line, ES2 following transfection with the CDKN1A siRNA SMARTpool. Silencing of CDKN1A expression results in dasatinib resistance. Each point represents the mean and SEM of six replicates. CDKN1A siRNA dasatinib survival curve vs. control siRNA two-way ANOVA $p<0.0001$ for OVISE and $p=0.02$ for ES2. (C) Dasatinib dose response curves for the ARID1A mutant OCCC cell line OVISE and the $A R I D 1 A^{\mathrm{Q} 456^{*} / \mathrm{Q} 456^{*}}$ isogenic clone following transfection with four individual CDKN1A siRNA oligos and the CDKN1A SMARTpool. Each of the individual siRNAs results in dasatinib resistance suggesting that the effect observed is not due to an off-target effect. For each CDKN1A siRNA dasatinib survival curve vs. control siRNA two-way ANOVA $p<0.001$ (D) Western blot demonstrating the on-target nature of the CDKN1A siRNA. OVISE cell line was transfected with CDKN1A siRNA and whole cell lysates collected 48 hours later. Lysates were probed with the p21 antibody and a fluorescent-dye labeled secondary antibody used.

Figure 6. In vivo assessment of ARID1A mutant xenograft models. (A) Representative IVIS luminescent images of mice with TOV21G peritoneal xenografts after 14 days dasatinib treatment. TOV21G cells were infected with a luciferase expressing lentivector and injected into the peritoneum of BALB athymic mice $(n=40)$. Twenty-four hours later, dasatinib (15 mg/kg/day, $n=20)$ or vehicle $(n=20)$ was initiated Ten minutes prior to IVIS imaging, mice were administered luciferin $(10 \mathrm{ul} / \mathrm{mg}$ ) intraperitoneally. Coloured bar represents photon count $/ \mathrm{cm}^{2} / \mathrm{sec}$. (B) Bar chart of mean luminescence values and SEM 
of photons $/ \mathrm{cm}^{2} / \mathrm{sec}$. Dasatinib treated vs. vehicle treated, two-way ANOVA $\mathrm{p}<$ 0.01 . 

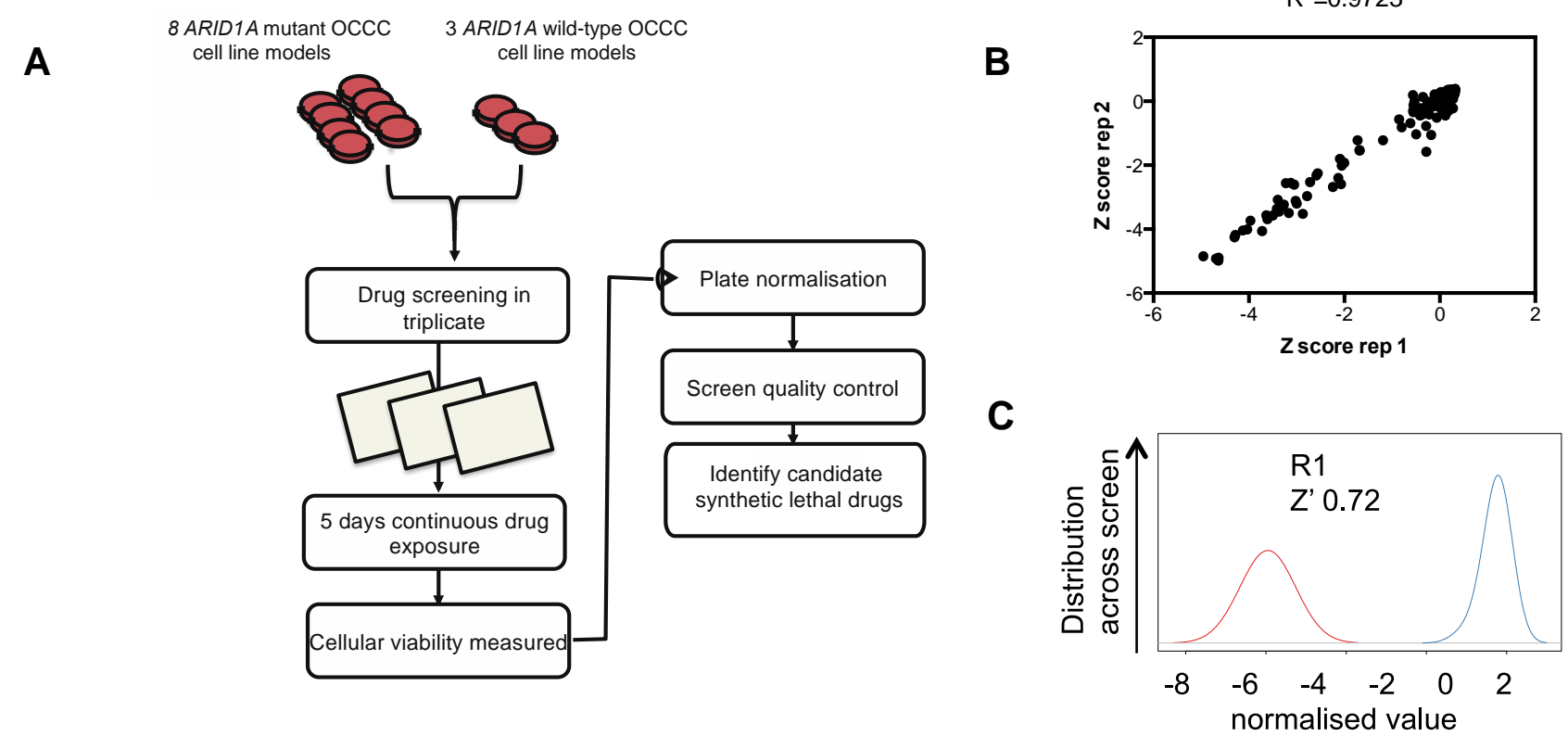

D

E
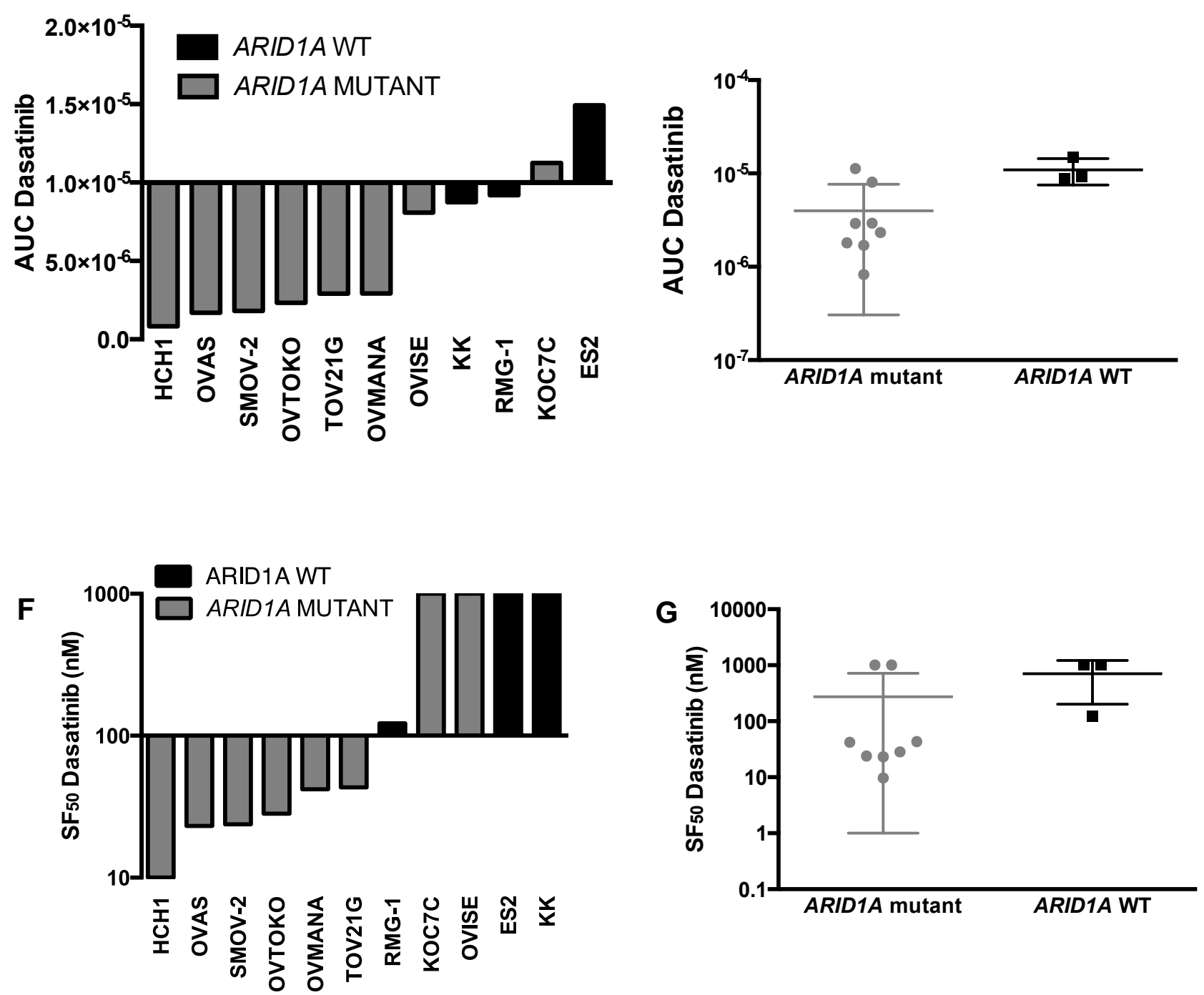
A

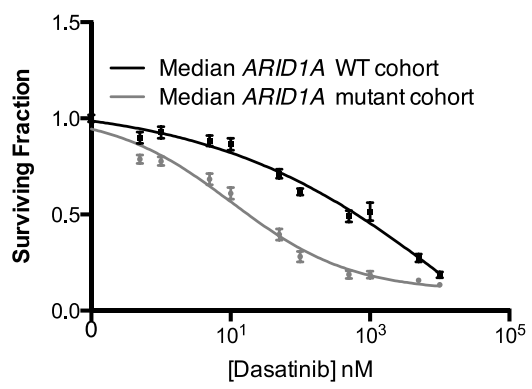

D

\section{DMSO Dasatinib $10 \mathrm{nM}$}
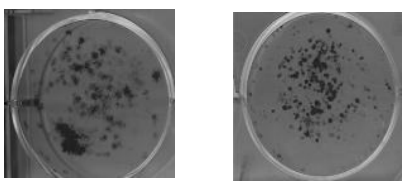

ARID1A wild type RMG-1
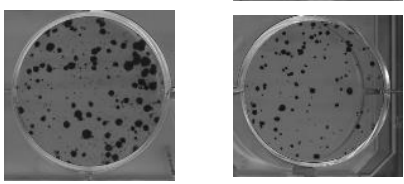

ARID1A wild type KK
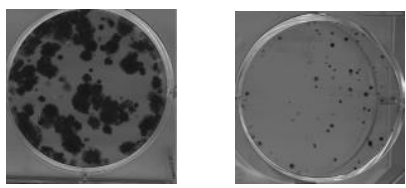

ARID1A mutant OVISE
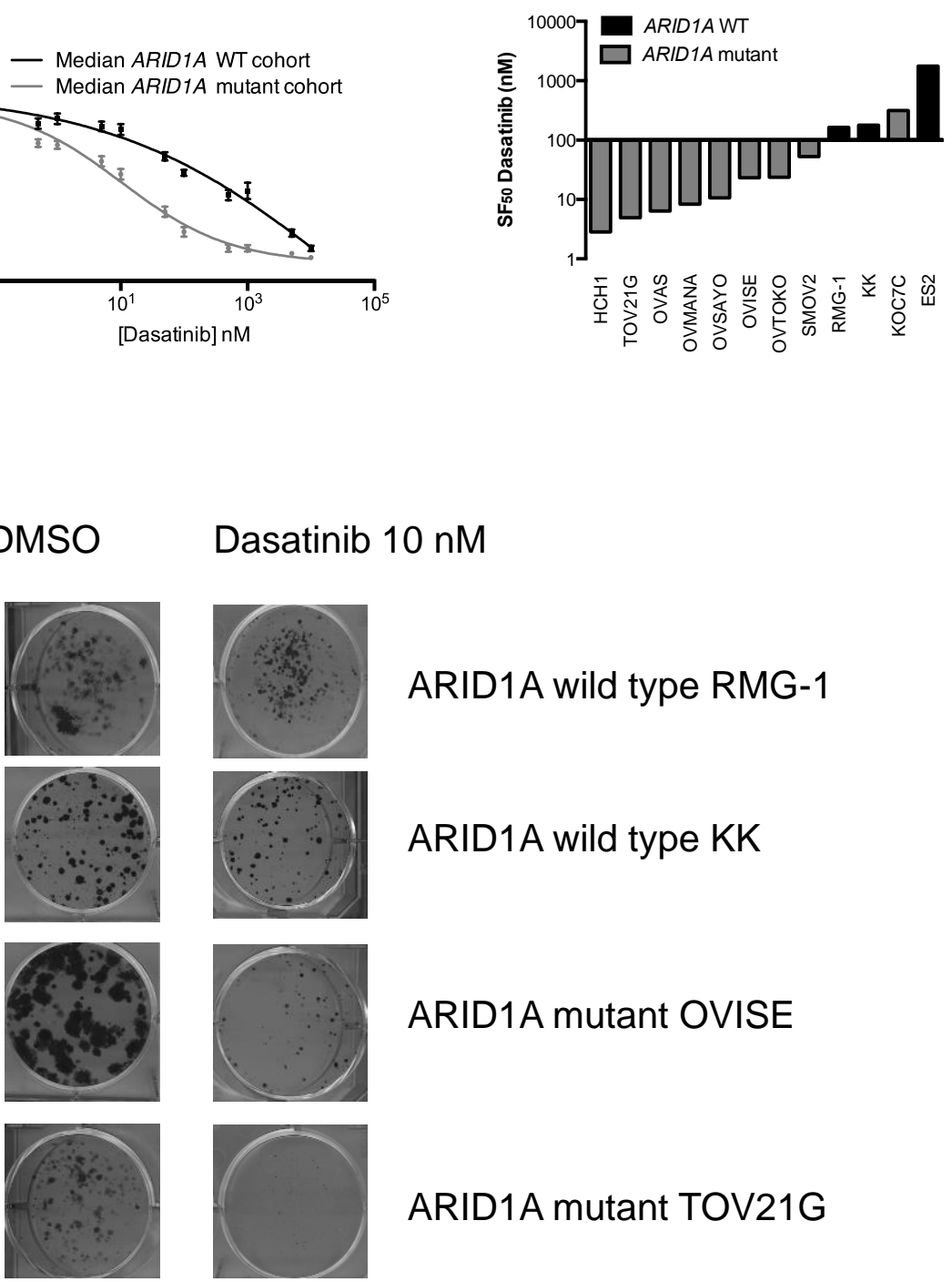

$\mathbf{F}$

ARID1A mutant TOV21G

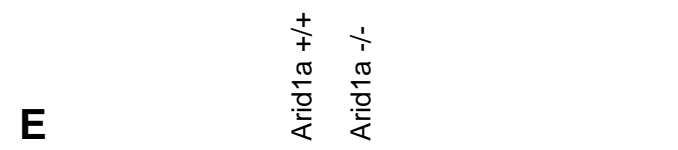

Arid1a

$250 \mathrm{kDa}$

$\beta$ Tubulin $40 \mathrm{kDa}$

Mouse ES cell line

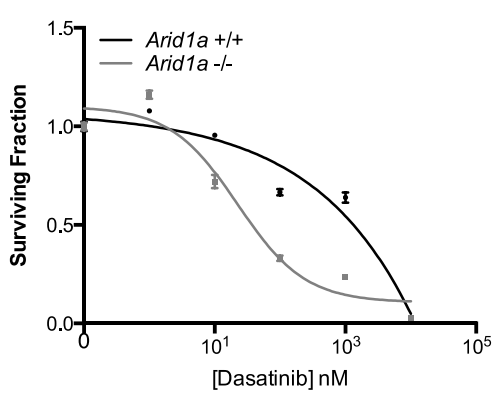

H

ARID1A

$250 \mathrm{kDa}$

$\beta T U B U L I N$

40kDa
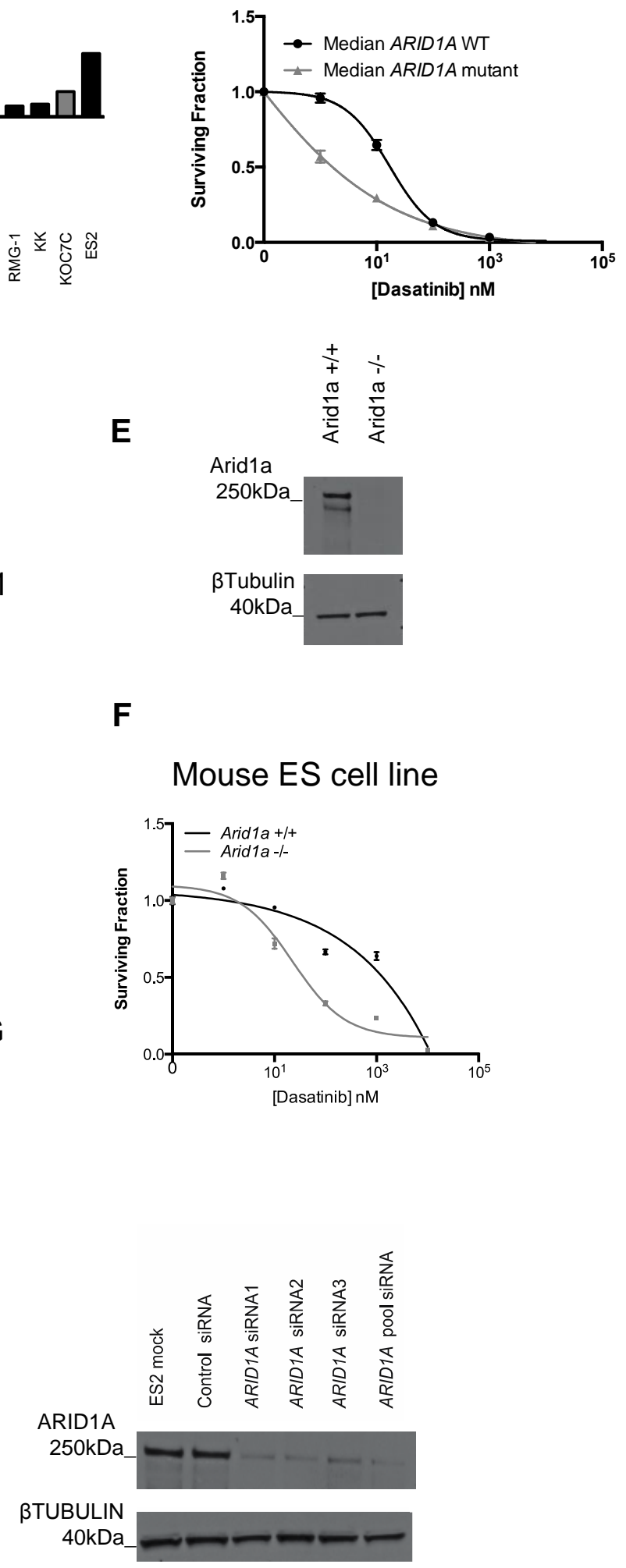

G

Human OCCC ARID1A wild type ES2

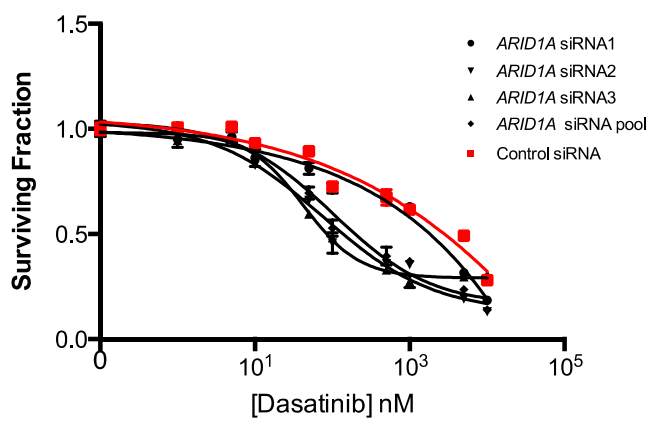


Figure 3

A

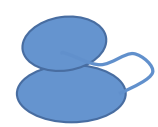

Inactive

Kinase
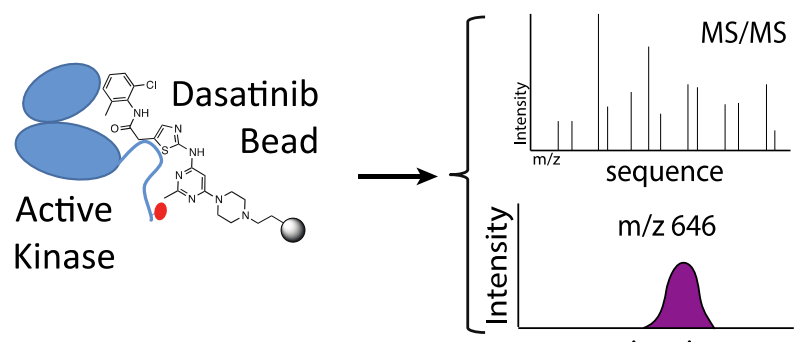

retention time
B

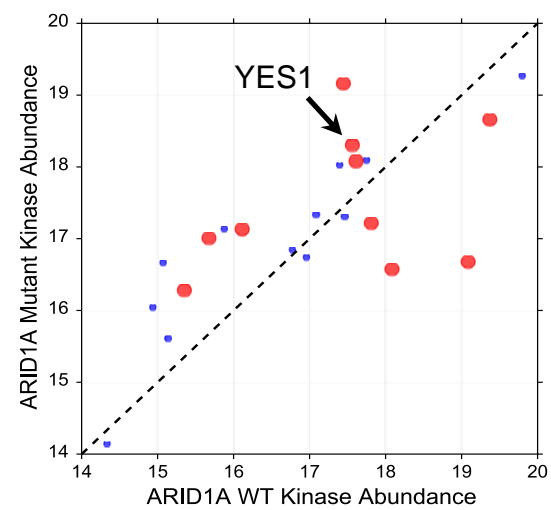

C
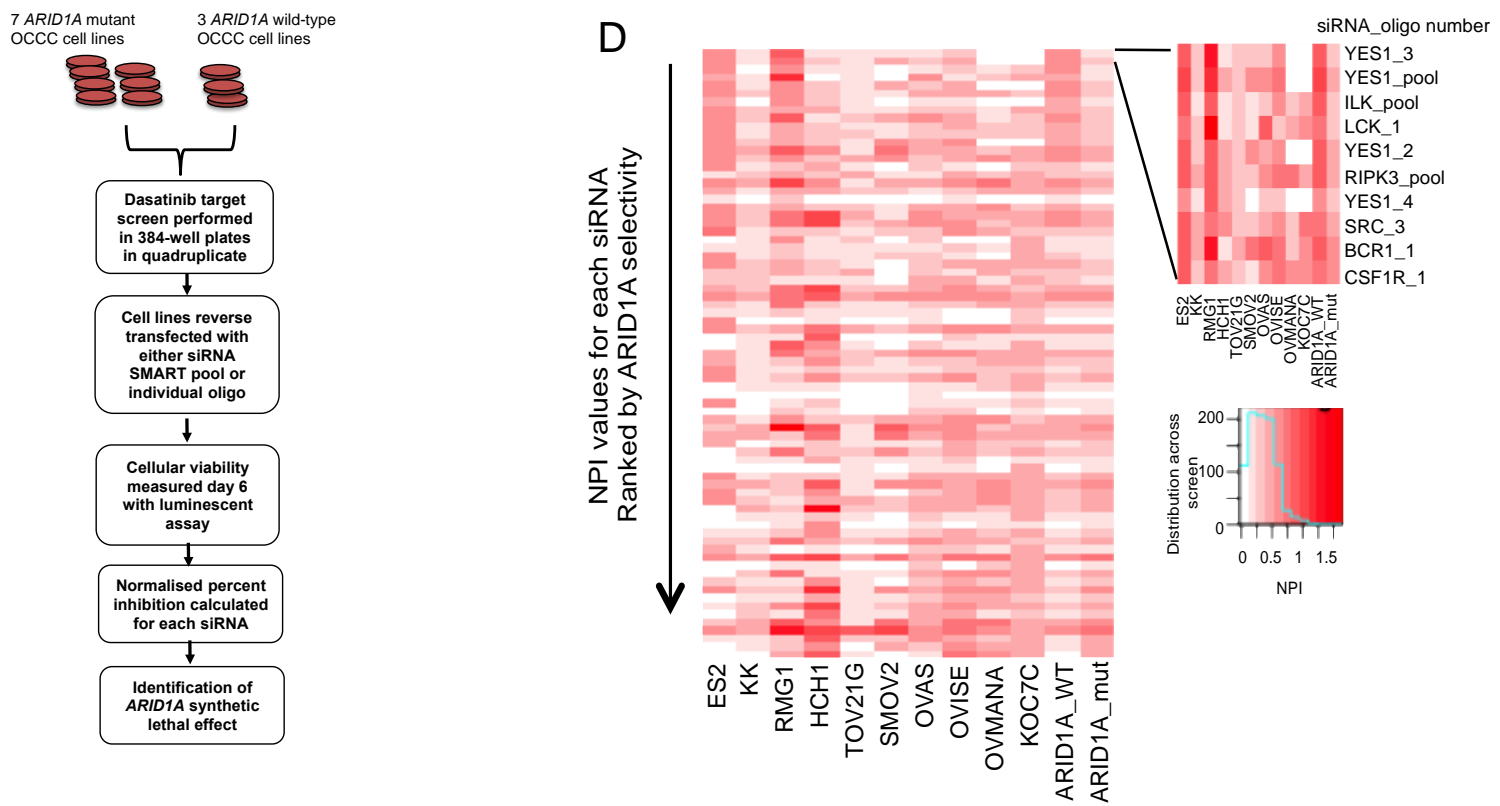

E

Median NPI ARID1A

F

YES1

wild-type cohort

$\square$ Median NPI ARID1A

mutant cohort
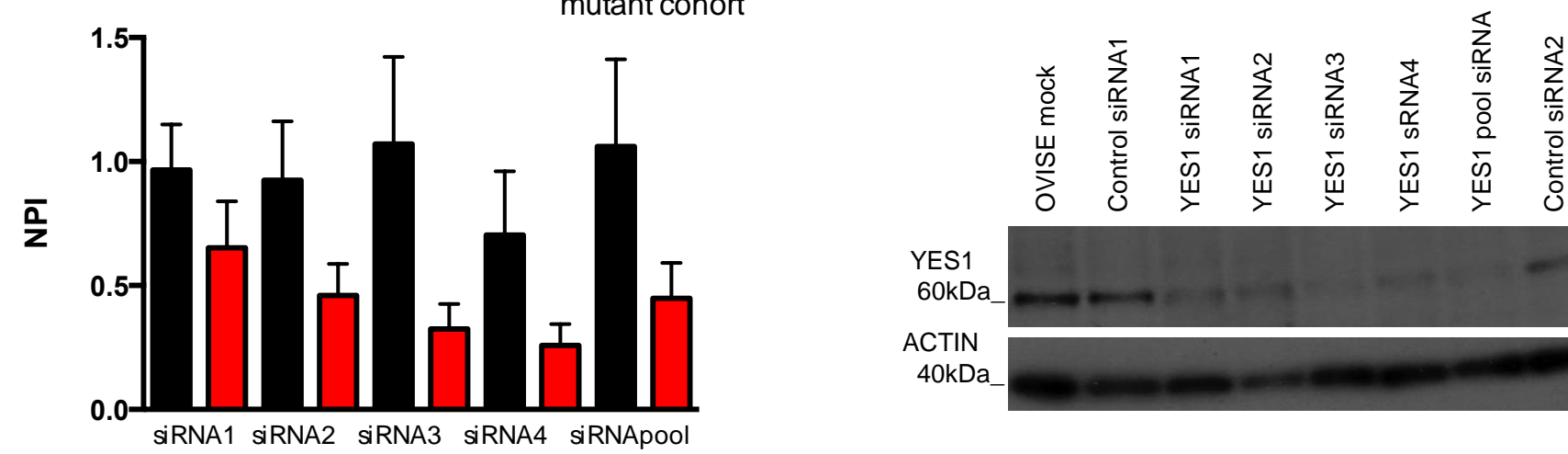

YES1

$60 \mathrm{kDa}$

ACTIN

$40 \mathrm{kDa}$ 
Figure 4

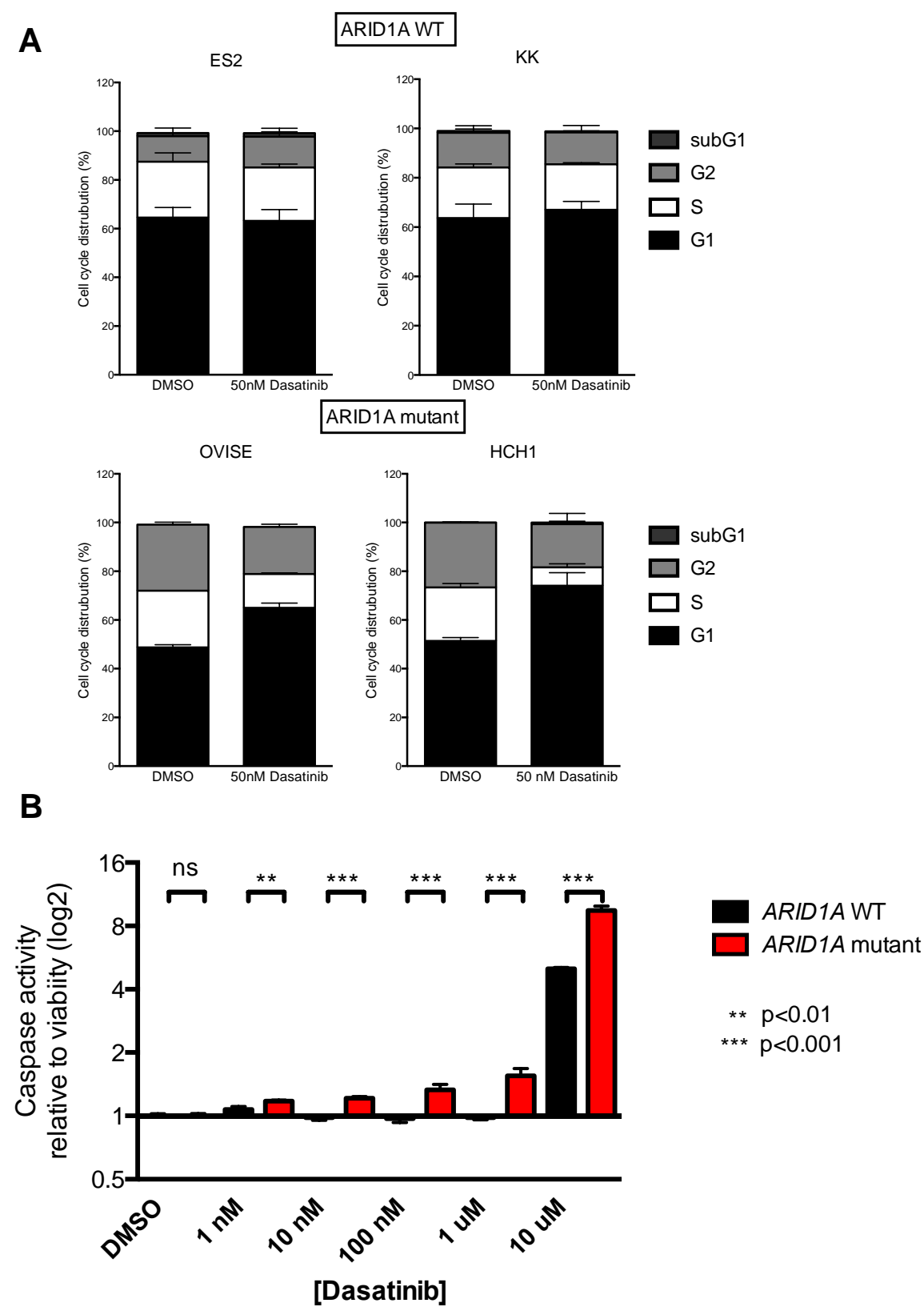


Figure 5

A
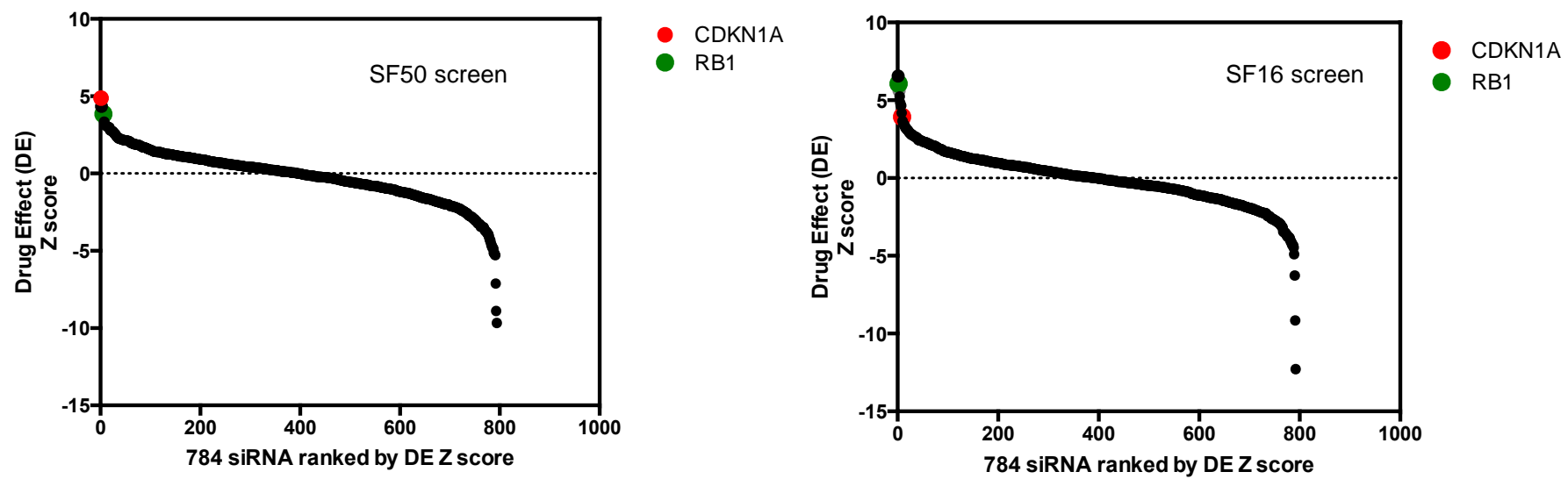

B
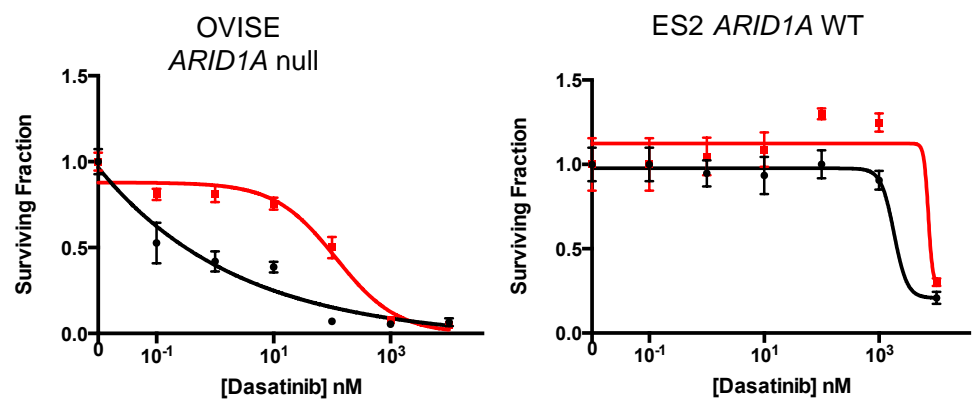

C

OVISE ARID1A null

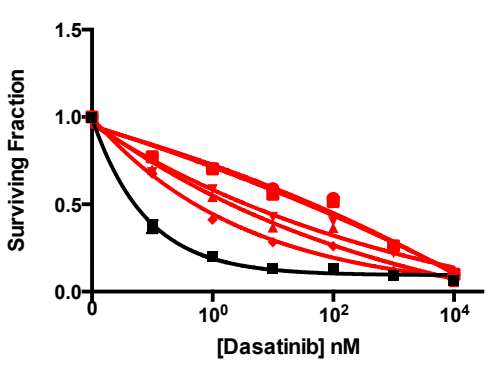

CDKN1A

$\mathrm{RB}$

CDKN1A

- CDKN1A siRNA pool

ARID1A Q456*/Q456*

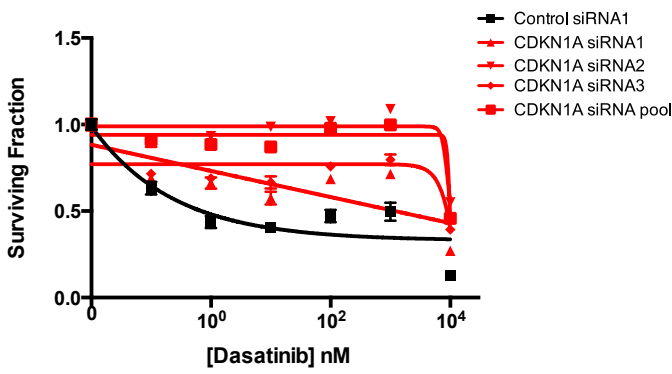

ACTIN

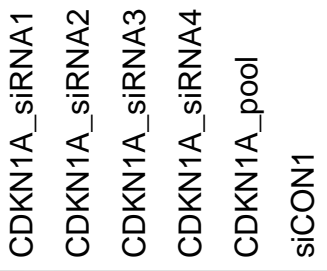

$40 \mathrm{kDa}$ 
Figure 6

A
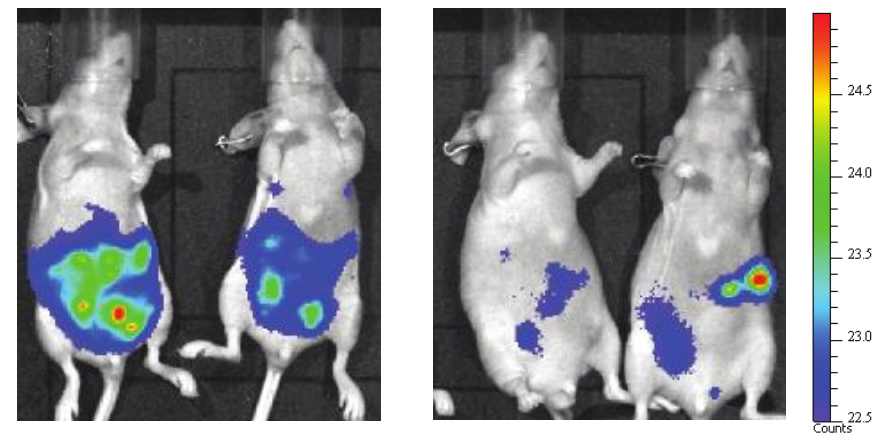

Vehicle

Dasatinib

B

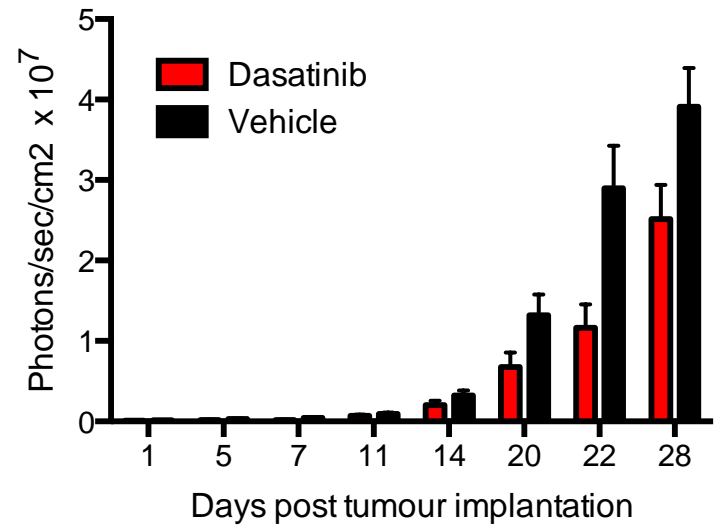

